\title{
Beam Coupling Impedance of Fast Stripline Kickers
}

\author{
B.R. Poole \\ G.J. Caporaso \\ Y.J. (Judy) Chen \\ S.D. Nelson
}

This paper was prepared for submittal to the

1999 Particle Accelerator Conference

New York, NY

March 29-April 2, 1999

March 1999

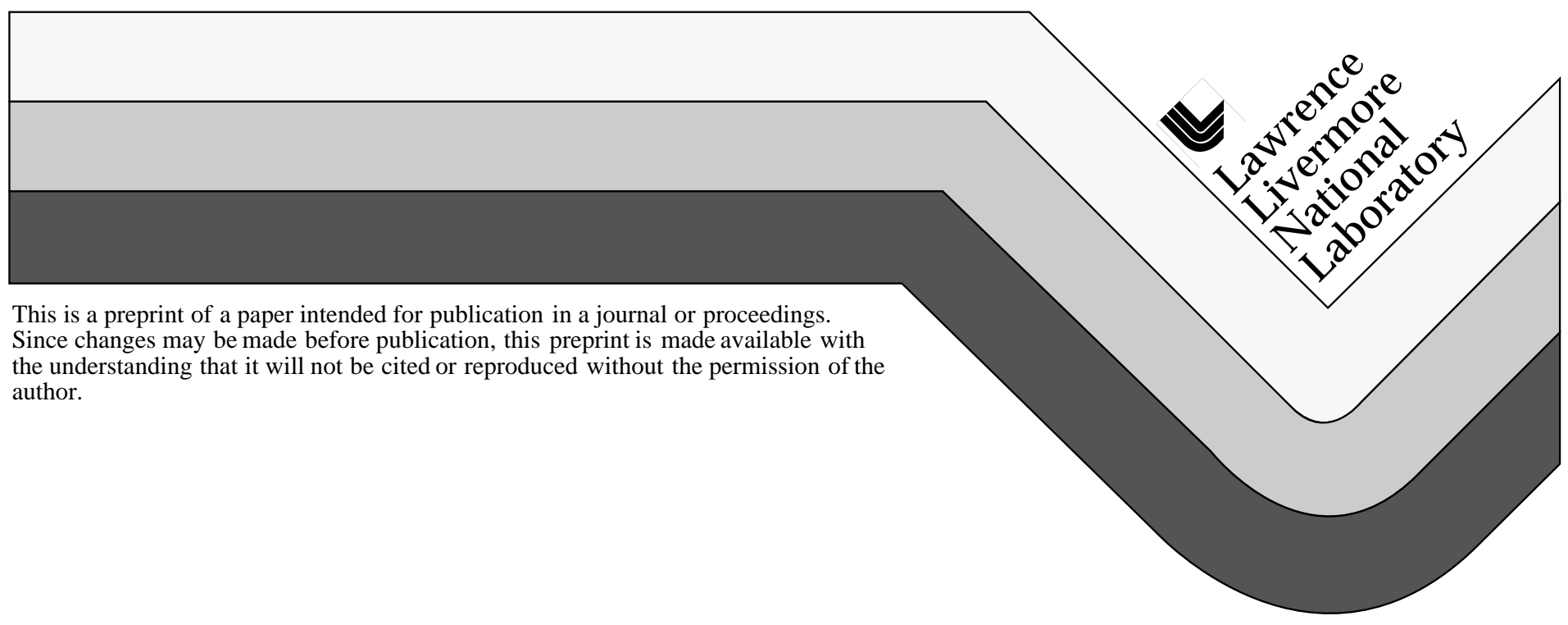




\section{DISCLAIMER}

This document was prepared as an account of work sponsored by an agency of the United States Government. Neither the United States Government nor the University of California nor any of their employees, makes any warranty, express or implied, or assumes any legal liability or responsibility for the accuracy, completeness, or usefulness of any information, apparatus, product, or process

disclosed, or represents that its use would not infringe privately owned rights. Reference herein to any specific commercial product, process, or service by trade name, trademark, manufacturer, or otherwise, does not necessarily constitute or imply its endorsement, recommendation, or favoring by the United States Government or the University of California. The views and opinions of authors expressed herein do not necessarily state or reflect those of the United States Government or the University of California, and shall not be used for advertising or product endorsement purposes. 


\title{
BEAM COUPLING IMPEDANCE OF FAST STRIPLINE BEAM KICKERS
}

\author{
B. R. Poole ${ }^{1}$, G. J. Caporaso, Y. J. (Judy) Chen, S. D. Nelson, LLNL, Livermore, CA
}

\section{Abstract}

A fast stripline beam kicker is used to dynamically switch a high current electron beam between two beamlines. The transverse dipole impedance of a stripline beam kicker has been previously determined from a simple transmission line model of the structure [1]. This model did not include effects due to the long axial slots along the structure as well as the cavities and coaxial feed transition sections at the ends of the structure. 3-D time domain simulations show that the simple transmission line model underestimates the low frequency dipole beam coupling impedance by about $20 \%$ for our structure. In addition, the end cavities and transition sections can exhibit dipole impedances not included in the transmission line model. For high current beams, these additional dipole coupling terms can provide additional beam-induced steering effects not included in the transmission line model of the structure [2], [3].

\section{INTRODUCTION}

The stripline kicker is designed to spatially separate a high current electron beam for transport into two separate beam lines. This system is used in conjunction with a static magnetic dipole septum to provide an additional angular kick to the beam. This allows the two beamlines to diverge sufficiently fast as to incorporate additional focusing elements for further downstream transport. This system is shown schematically in Fig. 1.
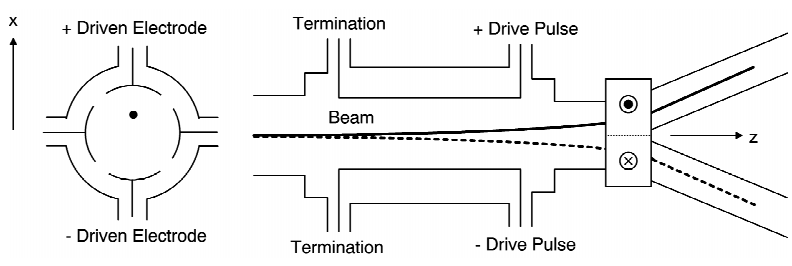

Figure 1: Stripline kicker and septum configuration

A high voltage pulse is applied to the downstream ports of the kicker and the beam is kicked by the electric and magnetic fields associated with the TEM waves propagating on the strip transmission lines. The beam is then directed into the septum magnet with opposite polarity dipole fields on either side of the plane separating the two downstream beamlines. All the upstream ports

"This work was performed under the auspices of the U.S. Department of Energy by the Lawrence Livermore National Laboratory under contract No. W-7405-Eng-48.

Email: poole1@1lnl.gov and the two downstream ports in the non-kick plane are terminated in a matched load impedance for the dipole transmission mode on the structure.

\section{KICKER TEM FIELDS}

To steer the beam in $x$, opposite polarity high voltage pulses are applied to the downstream ports in the $y=0$ plane. The potential within the kicker plates $(r<b)$ is given by

$$
V=\frac{4 V_{p}}{\pi} \sum_{n=o d d}\left(\frac{1}{n}\right) \sin \left(\frac{n \phi_{0}}{2}\right) \cos (n \phi)\left(\frac{r}{b}\right)^{n}
$$

where $b$ is the interior radius of the kicker plates, $\phi_{0}$ is the angle subtended by each kicker plate, and $V_{p}$ is the kicker plate voltage giving a total steering voltage of $2 V_{p}$. The solution is determined by solving for the potential in the interior region subject to the boundary condition that the applied potential at $r=b$ is given by the appropriate applied plate voltages and the potential in the gaps between the plates is zero. The $n=1$ term in Eq. 1 represents the transverse dipole force which provides the beam steering. The beam deflection due to the combined TEM electric and magnetic dipole forces is given by

$$
\Delta x=\frac{4 V_{p}}{\pi b} \sin \left(\frac{\phi_{0}}{2}\right)\left(\frac{q}{\beta \gamma m c^{2}}\right) \ell^{2}
$$

where $\ell$ is the length of the kicker.

\section{DIPOLE WAKE IMPEDANCE AND BEAM-INDUCED STEERING}

For intense beam applications, the beam current is sufficiently large as to induce substantial voltages and currents on the strip transmission lines. These TEM fields are introduced on the transmission lines as the beam traverses the upstream and downstream gaps as well as from changes in the dipole return current as the beam is deflected. These fields can than interact with the beam to cause beam-induced steering. In the limit of ultrarelativistic stiff beams the strength of this interaction is related to the dipole wake impedance.

\subsection{Dipole Impedance}

The $n=1$ transverse dipole wake impedance for the structure with an ultra-relativistic stiff beam centroid transported through the kicker structure offset form the $z$ axis by an amount $x_{0}$ is given by [1] 


$$
\begin{aligned}
Z_{\perp}(\omega)= & \frac{8 c Z_{k}}{\pi^{2} b^{2}} \sin ^{2}\left(\frac{\phi_{0}}{2}\right)\left(\frac{1}{\omega}\right)\left[\sin ^{2}\left(\frac{\omega \ell}{c}\right)\right. \\
& \left.+j \sin \left(\frac{\omega \ell}{c}\right) \cos \left(\frac{\omega \ell}{c}\right)\right]
\end{aligned}
$$

A kicker has been designed and installed on the Experimental Test Accelerator (ETA-II). The ETA-II kicker has the following set of parameters: $b=12.86 \mathrm{~cm}$, $R_{0}=19.5 \mathrm{~cm}, \phi_{0}=78^{\circ}, Z_{k}=50 \Omega$, and $\ell=160 \mathrm{~cm}$ where $R_{0}$ is the outer vacuum chamber radius. The structure was modelled using the LLNL 3-D time domain electromagnetic code, TIGER [4] to determine the dipole impedance. Figures 2 and 3 show a comparison of the real and imaginary parts of the dipole impedance as calculated from Eq. 3 with the numerical results from the 3-D electromagnetic code.

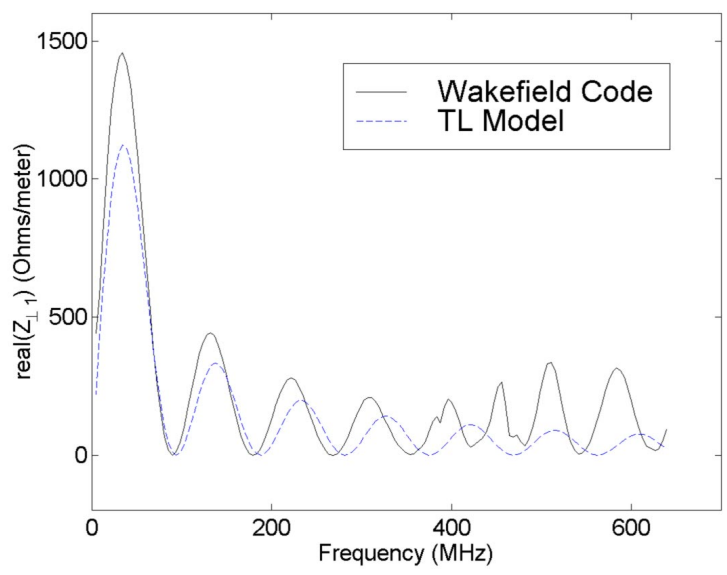

Figure 2: Real part of dipole impedance

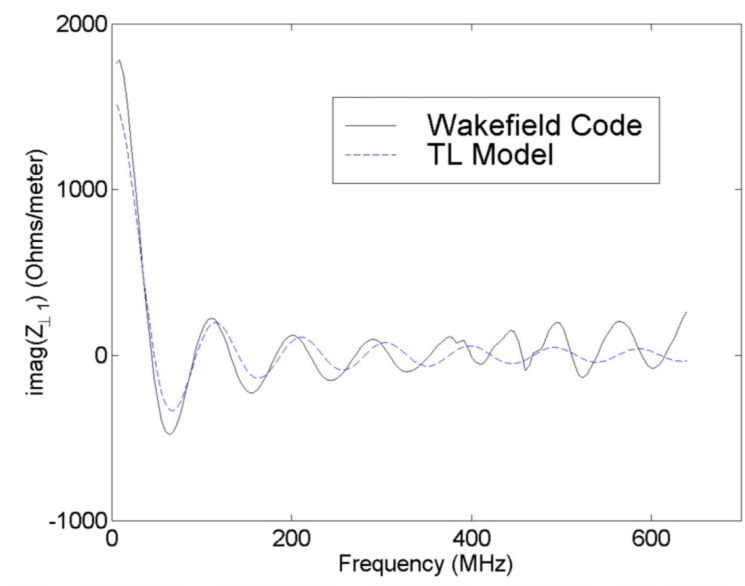

Figure 3: Imaginary part of dipole impedance

As can be seen in Figures 2 and 3, there is a good agreement between the transmission line model and the 3-D code results in the low frequency regime. The differences become more pronounced at higher frequencies as the end cavity transition sections associated with the feeds to the external $50 \Omega$ coaxial ports play a more significant role in the coupling. More will be discussed about this in section 4 .

\subsection{Beam Induced Steering}

A detailed model of beam-induced steering has been previously described [2], [3]. The centroid motion of the beam in the kicker is given by [5]

$$
\begin{gathered}
\left(\frac{\partial}{\partial z}+\frac{s}{c}\right)^{2} x(z)=\frac{V_{p}}{V_{0}} e^{-\frac{s}{c}(\ell-z)} \\
+\frac{2 I_{b}}{I_{c} \ell^{2}}\left[x(z)-2 \frac{s}{c} e^{\frac{s}{c} z} \int_{z}^{\ell} x\left(z^{\prime}\right) e^{-\frac{s}{c} z^{\prime}} d z^{\prime}\right]
\end{gathered}
$$

where $s$ is the Laplace transform variable, $\partial / \partial t \rightarrow s$, and $I_{b}$ is the beam current. The critical current, $I_{c}$ is given by

$$
I_{c}=\frac{\pi}{16}\left(\frac{b}{\ell}\right)^{2} \frac{Z_{0}}{Z_{k}} \frac{\gamma \beta^{2} I_{0}}{\sin ^{2}\left(\phi_{0} / 2\right)}
$$

and the characteristic voltage, $V_{0}$ is defined as

$$
V_{0}=I_{c} Z_{k} \frac{\ell}{2 \pi b} \sin \left(\frac{\phi_{0}}{2}\right)
$$

where $I_{0}=17 \mathrm{kA}$ and $Z_{0}=377 \Omega$. Equation 4 can in principle be solved numerically for a particular set of conditions. Of particular interest is the case of an initially offset beam entering the kicker structure parallel to the beamline axis without any applied voltage since this solution can be related to the dipole wake impedance. It has been shown previously [3], that the asymptotic beam deflection for an initially offset beam has the form

$$
x(z=\ell, t \rightarrow \infty)=x_{0} \cosh \left(\sqrt{\frac{2 I_{b}}{I_{c}}}\right)
$$

where $x_{0}$ is the injection offset of the beam. For sufficiently small beam currents the asymptotic deflection is related to the dipole wake impedance by

$$
x(z=\ell, t \rightarrow \infty) \approx x_{0}\left[1+\left(\frac{2 \pi \ell}{\gamma \beta^{2} I_{0} Z_{0}}\right) I_{b} Z_{\perp 0}\right]
$$

where $Z_{\perp 0}=\operatorname{Im}\left[Z_{\perp}(\omega=0)\right]$. By numerically integrating Equation 4 it is possible to find a temporal solution for the beam displacement of the beam leaving the kicker. Consider an ETA-II electron beam with $6 \mathrm{MeV}$ energy and a $2 \mathrm{kA}$ beam current with a $1 \mathrm{~cm}$ initial offset. For these parameters, the critical current, $I_{c}$ is about $4.2 \mathrm{kA}$ and the asymptotic deflection given by Eq. 7 is $1.51 x_{0}$. Figure 4 shows the temporal evolution of the beam displacement leaving the kicker from numerical integration of Eq. 4. Figure 4 shows a quite dramatic beam-induced steering effect for a high current beam. The time scale for reaching the asymptotic value is on the 
order of the round-trip transit time of the structure, $2 \ell / c \sim 10.6 \mathrm{~ns}$.

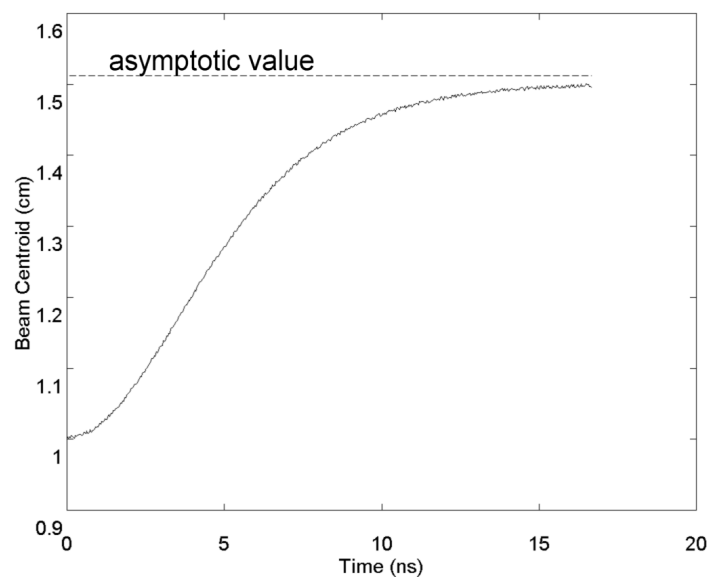

Figure 4: Temporal variation of beam centroid exiting ETA-II kicker for initial offset of $1 \mathrm{~cm}$

\section{CORRECTIONS TO THE DIPOLE IMPEDANCE FROM THE CAVITY TRANSITION SECTIONS}

The 3-D code results show that the simple transmission line model underestimates the low frequency dipole wake impedance. Measurements of the kicker impedance show that the structure is not very well matched over a wide band of frequencies. In an effort to obtain a correction to the impedance at low frequencies, a simple inductance was incorporated as a model of the transition section connecting the strip transmission lines to the external coaxial ports. It was found that a $40 \mathrm{nH}$ inductor would given an input impedance consistent with the experimental data. Figure 5 shows a plot of the experimental data and the projected low frequency input impedance.

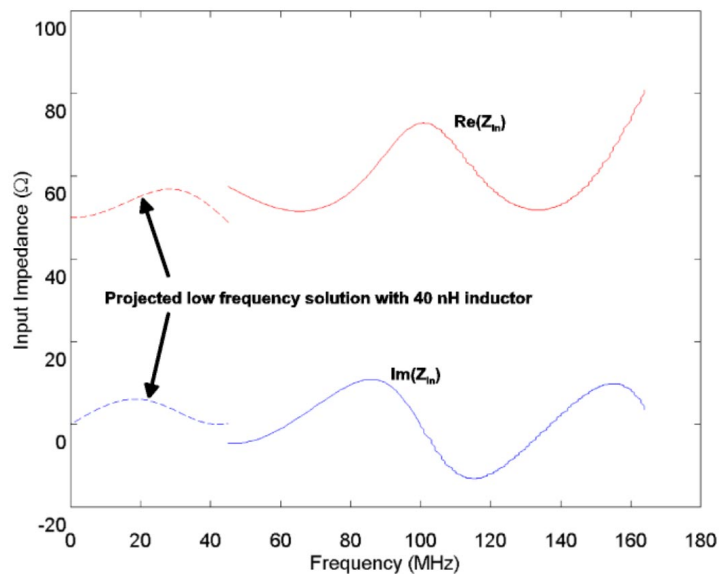

Figure 5: Kicker input impedance

The kicker dipole impedance is calculated including the series inductors to model the transition sections. Figures 6 and 7 show the real and imaginary parts of the dipole impedance for low frequencies.

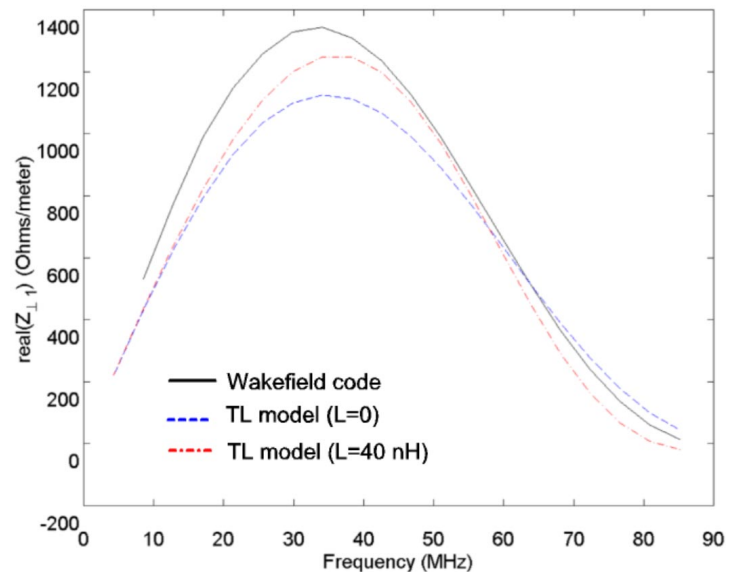

Figure 6: Real part of wake impedance with transition

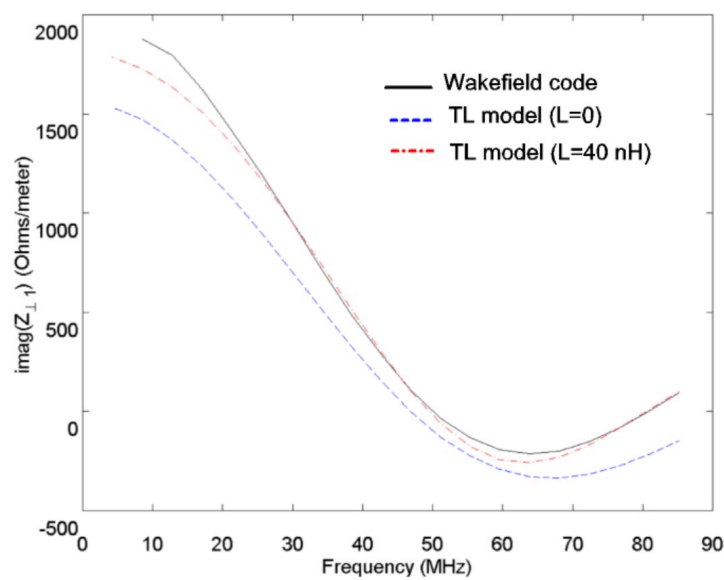

Figure 7: Imaginary part of dipole impedance with transition

As can be seen the transitions can modify the dipole wake impedance of the structure. In principle, additional reactive elements can be incorporated to extend the model to higher frequencies.

\section{REFERENCES}

[1] K. Y. Ng, "Impedances of Stripline Beam-Position Monitors," Particle Accelerators 23, 93-102 (1988)

[2] G. J. Caporaso, Y. J. Chen, B. R. Poole, "Transmission Line Analysis of Beam Deflection in a BPM Stripline Kicker," 1997 Particle Accelerator Conference, Vancouver, B. C. Canada, May 12-16, 1997

[3] B. R. Poole, G. J. Caporaso, Y. J. Chen, L. F. Wang, "Analysis and Modelling of a Stripline Beam Kicker and Septum,” XIX International Linac Conference, Chicago, Ill, USA, August 23-28, 1998

[4] D.Steich, J. Kallman, J.B. Grant, D. Mayhall, B. Poole, K. Kunz, H. Brand, G. Laguna, "TIGER: An Object Oriented Time-Domain Electromagnetics Simulation Code," LLNL Thrust Area Report, Computational Electronics and Electromagnetics, sec. 1., pp. 13-20, UCRL-ID-125470, 1997

[5] Y. J. Chen, "Fast Kicker for High Current, Continuous Beams," To be published 